\title{
Evaluation of idiopathic epiretinal membranes by a scanning laser ophthalmoscope
}

\author{
Yuichiro Ogura, Yoshihito Honda
}

A scanning laser ophthalmoscope provides real time retinal images of high contrast with the use of confocal laser optics. Previous studies have shown that the scanning laser ophthalmoscope is useful for fluorescein angiography, ophthalmoscopy with a low amount of light, retinal perimetry, and localised retinal acuity testing. ${ }^{1-3}$ We applied the scanning laser ophthalmoscope for an evaluation of patients with idiopathic epiretinal membranes before vitreous surgery.

\section{Case report}

A 57-year-old man was referred with a diagnosis of the epiretinal membrane in the left eye. He has noticed blurred vision and a metamorphopsia in the left eye for several years. His visual acuity was 20/20 right eye and 20/80 left eye. The epiretinal membrane at the macula was observed with conventional ophthalmoscopy (Fig 1). The fundus images by the scanning laser ophthal-

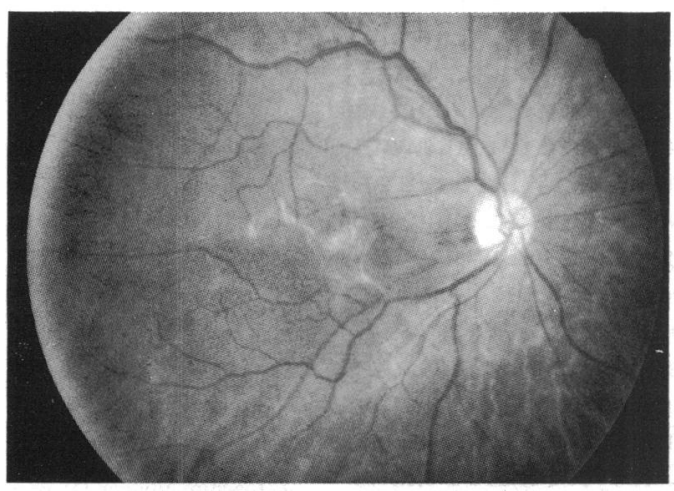

Figure 1 Fundus photograph with a conventional fundis camera. Note the epiretinal membranes over the macula.

\footnotetext{
Department of Ophthalmology, Kyoto University Faculty of Medicine, Kyoto, Japan Y Ogura Y Honda

Correspondence to: Yuichiro Ogura, MD, Department of Ophthalmology, Kyoto University Faculty of Medicine, Sakyo-ku, Kyoto 606, Japan.

Accepted for publication 19 March 1993
}

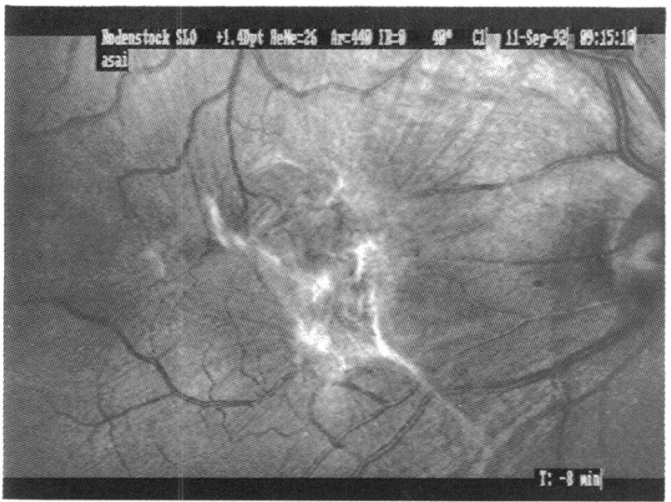

Figure 2 Fundus photograph with a scanning laser ophthalmoscope with argon laser illumination. The epiretinal membrane and changes in the retinal surface adjacent to the membrane are well visualised.

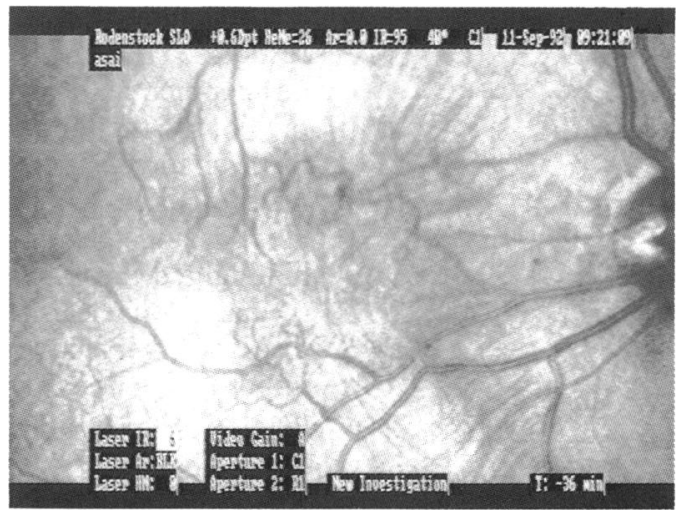

Figure 3 Fundus photograph with a scanning laser ophthalmoscope and infrared laser illumination. The details of the retina under the epiretinal membrane are well assessed.

moscope (Rodenstock Instrument, Munich, Germany) with argon laser illumination (418 and $514 \mathrm{~nm}$ ) clearly demonstrated the epiretinal membrane over the macula (Fig 2). Since the argon laser beam of a short wavelength is reflected and scattered by the surface of the epiretinal membrane, the extent of the membrane was confirmed. Radiating retinal folds around the membrane were also well visualised. Additionally, it was possible to make a rough estimate of the thickness of epiretinal membranes using the confocal facility of the scanning laser ophthalmoscope. The images taken with the infrared laser illumination $(780 \mathrm{~mm})$ revealed the details of the macular retina and choroid under the epiretinal membrane (Fig 3). Changes in the retinal vessels caused by the traction due to the epiretinal membrane were well recognised. The patient underwent vitrectomy and peeling of the epiretinal membrane. The membrane was successfully removed and his visual acuity has improved to $20 / 40$.

\section{Comment}

This report demonstrated that an examination of the fundus by the scanning laser ophthalmoscope is useful for an evaluation of patients with epiretinal membranes. The images with the blue argon laser illumination clearly showed the extent of the membrane and changes in the retinal surface adjacent to the membrane by enhanced scatter and reflection of this wavelength. On the other hand, an observation with the infrared laser beam of the longer wavelength provided images of the underlying retina and choroid by the enhanced tissue penetration of this wavelength. Unlike the conventional optical systems, the scanning laser ophthalmoscope could visualise structures of the fundus with high 
contrast even by illumination with the long wavelength. These features of the scanning laser ophthalmoscope allow an assessment of abnormal vitreoretinal interfaces such as epiretinal membranes.
1 Webb RH, Hughes GW, Delori FC. Confocal scanning laser ophthalmoscope. Applied Optics 1987; 26: 1492-9.

2 Mainster MA, Timberlake GT, Webb RH, Hughes GW. Scanning laser ophthalmoscopy. Clinical applications. Ophthalmology 1982; 89: 852-7.

3 Wolf S, Arend O, Toonen H, Eng D, Bertram B, Jung F, et al Retinal capillary blood flow measurement with a scanning laser ophthalmoscope. Ophthalmology 1991; 98: 996-1000. 